\title{
EFFECT OF SURFACE ORIENTATION OF THE SUBSTRATE ON THE SATURATION STATE OF SOLUTION DURING LIQUID PHASE HETEROEPITAXY
}

\author{
J.M. OLCHOWIK \\ Technical University of Lublin, Department of Physics \\ Nadbystrzycka 38, 20-618 Lublin; Poland
}

\begin{abstract}
Orientation effects during heteroepitaxy appear at the stage of the layer formation and also during the isothermal contact of the multicomponent solution with the binary substrate. In the paper, the analysis of the $\operatorname{In} P$ and $\mathrm{GaP}$ substrate orientation on the state of liquid phase was carried out. The analysis based on the in situ comparative determination of the contact supersaturation of the Ga-In-P-As and Ga-In-P solutions. It was found that the contact supersaturation of the alloy was connected with the coupling mechanism of the interface with the binary substrate.
\end{abstract}

PACS numbers: 68.55.-a

\section{Introduction}

Crystallographic orientation of the substrate effects the composition of the liquid phase formed by the substrate-source method and also effects the parameters of synthesized layers $[1,2]$. However, the published data usually describe the crystallization process only and do not provide any information regarding the phenomena appearing during solution formation. It is acknowledged that the contact of the multicomponent liquid solution with the binary substrate leads to the formation of the transition layer. The interphase range introduces therefore a contribution to the energetic balance of the system and it depends mostly on the parameters of the solid phase surface. In the paper, the solubility of binary GaP and InP substrates of various crystallographic orientation in the liquid Ga-In-P and Ga-In-P-As phases during heteroepitaxy was analysed to prove the existence of the orientation effects. The liquid phase was formed either to match the liquid solution equivalent lattice to the substrate lattice or to make them different.

The value of the contact solution supersaturation was the criterion applied to estimate the effect of substrate orientation on the condition of liquid phase. The novel design cassette was used in the vertical variant of liquid phase epitaxy and the process was carried by the method [3] enabling to maintain the identical 
conditions during the contact of liquid phases with variously oriented crystalline substrates. The cassette, schematically depicted in Fig. 1, enabled to divide the initial solution into two portions at the liquidus temperature and then to bring them separately into the contact with two variously oriented substrates. The degree of the solution supersaturation caused by the contact with the substrates was estimated by the method described elsewhere [4].

\section{Experimental}

The $5 \mathrm{~g}$ portion of the solution formed according to the phase diagram was contained in the solution part (2) of the upper slider (1) (Fig. 1). The quartz tube

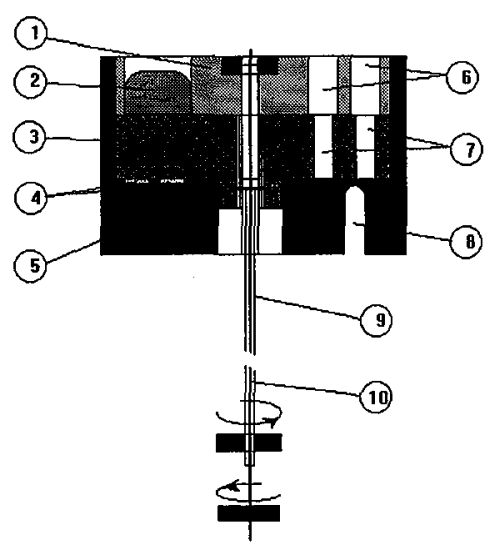

Fig. 1. Rotary cassette for comparative measurements of orientation effects.

surrounding the cassette (5) was purged with the purified hydrogen and heated to the temperature $20 \mathrm{~K}$ above the liquidus temperature. The homogenization time did not exceed 30 min under these circumstances. The point of the thermodynamical balance was in situ found observing a formation of homogeneous nuclei during temperature drop and their decay during the temperature rise.

The temperature cycles were applied a few times varying the temperature change rates, the liquidus temperature was considered being equal to the value obtained by the extrapolation of the nuclei formation temperatures (or their decay temperatures) to the zero temperature change rate.

The initial solution was then divided into the equal $2 \mathrm{~g}$ portions revolving the upper slider at the saturation temperature. These solution portions filled the solution containers (7) of the upper slider (3). The piping window (6) of the slider (1) was then' shifted above the containers filled with the solution and the containers were moved above the two substrates having different surface orientations. The axially positioned quartz rod (10) and pipe (9) enabled to change the relative positions of the substrates and solutions. The contacting phases were separated after the exposure which lasted depending on their interaction from a few to a few tens of minutes and the liquidus temperature of both solutions were again in situ determined. The points of thermodynamical equilibrium were found as for the 
initial solution. The above described method of the comparative investigations of the orientation effects during heteroepitaxy excludes the possible errors caused by the experimental difficulties in maintaining the same conditions in the series of experiments.

It is important, for the practical reasons, to know the orientation effects in the liquid phase - binary substrate systems in the range of compositions devoid of relaxational effects [5]. For this reason, the effects were analysed for the range of compositions of liquid solutions equivalent to the $\mathrm{Ga}_{x} \operatorname{In}_{1-x} \mathrm{P}$ solid phase for $x>0.7$. The starting temperatures of the analysis were chosen being equal to $T=$ $1073 \mathrm{~K}$. At this temperature, the coupling of the liquid phase with the $\mathrm{GaP}\langle 111\rangle$ and $\mathrm{GaP}\langle 100\rangle$ substrates can be considered coherent [5]. For the InP substrates, the experiment was carried out on the Ga-In-P-As solutions equivalent to the solid phase with the energetic gap corresponding to $\lambda=1.3 \mu \mathrm{m}$ for good lattice matching. The analysis was carried out at the solution saturation temperatures of $T=923 \mathrm{~K}$ and at the temperatures differing from the equilibrium temperature after the exposure.

\section{Results}

The in situ measured values of the contact supersaturation of the Ga-In-P solution after the isothermal contact with the $\mathrm{GaP}\langle 111\rangle$ and $\mathrm{GaP}\langle 100\rangle$ substrates are shown in Fig. 2a. For the coherent coupling of the liquid and solid phases [5], the latter receives the additional energy resulting from the deformation of the interphase. The energy balance can be written for one mole of the solution as

$$
\begin{aligned}
& R T \ln \gamma_{i j}^{\mathrm{s*}}=\frac{\partial}{\partial n_{i j}}\left(G^{\mathrm{el}} n+G^{\mathrm{ex}} n\right), \\
& \frac{1}{R T}\left(H_{i j}^{F}+T \Delta S_{i j}^{F}\right)+\ln \frac{\alpha_{i}^{\mathrm{l}} \alpha_{j}^{\mathrm{l}}}{\alpha_{i}^{\mathrm{sl}} \alpha_{j}^{\mathrm{sl}}}=\ln \alpha_{i j}^{\mathrm{s} *},
\end{aligned}
$$

where $\gamma_{i j}^{5 *}$ - the activity coefficient of the $i j$-th components at the interface, $H_{i j}^{F}, \Delta S_{i j}^{F}$ - enthalpy and entropy of the $i j$-th components, $\alpha_{i(j)}^{1}, \alpha_{i(j)}^{\mathrm{sl}}, \alpha_{i j}^{\mathrm{s*}}-$ the interatomic interaction parameters in liquid (l), stoichiometric liquid (sl) and solid ( $\left.\mathrm{s}^{*}\right)$ phases, $R$ - the gas constant, $G^{\mathrm{el}}$ - the elastic energy of the interface, $G^{\text {ex }}$ - the free mixing energy of the solid phase, $n_{i j}$ - the mole numbers of $i j$-th components in solid solution.

Taking into account the symmetry type of the $\mathrm{A}^{\mathrm{III}} \mathrm{B}^{\mathrm{V}}$ substrates and the crystallographic orientation of the interface, the formula (2) can be rewritten as

$$
R T \ln \gamma_{i j}^{\mathrm{s} *}=f\left(\alpha_{i j}^{\mathrm{s}}, x, y\right) ;
$$

and [5]

$$
R T \ln \gamma_{i j}^{\mathrm{s} *}=\sigma\left(a-a_{\mathrm{s}}\right)\left(2 a_{i j}-a-a_{\mathrm{s}}\right) ;
$$

where $\sigma$ - the parameter depending on the elastic constants of the solid phase, $a_{\mathrm{s}}$ - the lattice constant of the substrate, $a_{i j}$ - the lattice constant of the interface in coherent coupling, $a$ - the lattice constant of the interface in the free state.

The diagram of phase equilibrium with the included effect of surface deformation enables to forecast the change in the state of liquid phase at quasi-equilibrium 
during the coherent coupling of phases. The lattice deformation energy causes additional etching of the substrate [1] as the liquid phase and substrate form the isolated system. The separation of the substrate and solution produces the change in the liquidus temperature. This change also called the contact supersaturation of the solution is determined by the parameters of the substrate surface during the exposition.

The curves 1 and 2 in Fig. 2a were obtained from the diagram of the phase equilibrium including the effect of intersurface deformation. The experi-

[a]

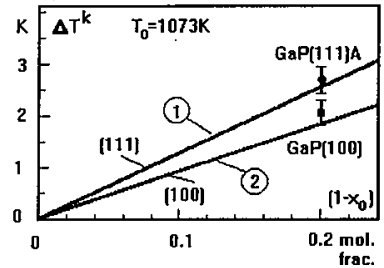

[b]

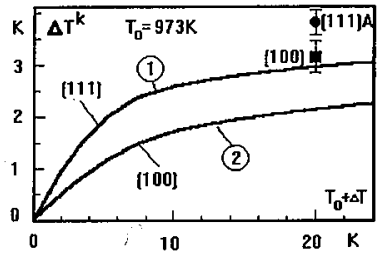

Fig. 2. Contact supersaturation of Ga-In-P (a) and Ga-In-P-As (b) solutions.

ment proved the stronger tendency of $\langle 111\rangle$ oriented substrates to isothermically supersaturate the solution and the qualitative convergence with the model of the coherent coupling of the phases $[5,8]$. The differences in the values are probably due to the fact that the energetic parameters disturbing solution homogeneity were omitted in the energetic balance.

Any significant differences in the values of contact supersaturation of the solution were not observed for the isoperiodic composition of the Ga-In-P-As/InP system. The effect of surface orientation on $\Delta T^{k}$ appeared only for exposure temperatures largely differing from the thermodynamical equilibrium. The in situ observed liquidus temperatures of this system (see Fig. 2b) are similarly in fair agreement with the theoretical calculations.

\section{References}

[1] Yu.B. Bolkhovityanov, Prog. Cryst. Growth Charact. 19, 159 (1989).

[2] H. Beneking, Cryst. Prop. Prep. 35, 1991.

[3] J.M. Olchowik, Polish Patent Nr P-293 606/92-02-24.

[4] V.V. Kuznetsov, J.M. Olchowik, V.S. Sorokin, Electr. Tech. ser. Mater. 4, 215 (1986).

[5] V.V. Kuznetsov, P.P. Moskvin, J.M. Olchowik, V.S. Sorokin, Inorg. Mater. 22, 11 (1986).

[6] V.V. Kuznetsov, P.P. Moskvin, V.S. Sorokin, Nierounoviesnyie iavlenia pri zidkostnoi heteroepitaksii poluprovodnikovykh tviordykh rastvorov, M. Metalurgia, 1991.

[7] A.S. Jordan, M. Illegems, J. Phys. Chem. Solids 36, 4 (1975).

[8] C.A.B. Ball, J.H. van der Merwe, in: Applications and Recent Advances, in series Dislocations in Solids, Vol. 6, Ed. F.R.N. Nabarro, North-Holland, Amsterdam 1983, p. 121. 